\title{
Physical vapor deposition of peptide nanostructures
}

\author{
Becky Bank-Srour ${ }^{1}$, Peter Becker ${ }^{1}$, Leonid Krasovitsky ${ }^{1}$, Alexander Gladkikh ${ }^{2}$, Yuri Rosenberg ${ }^{2}$, \\ Zahava Barkay $^{2}$ and Gil Rosenman ${ }^{1}$
}

Biological units such as proteins and peptides have the intrinsic ability to self-assemble into natural biological nanostructures such as DNA strands, proteins and amyloid fibers. These assemblies have become an inspiration for development of new supramolecular nanostructural materials self-assembled from chemically synthesized peptides and proteins. Bioinspired materials are in the center of interest in research and development, as they are considered as a new generation of functional soft materials, which can be used in a variety of applications. Peptide nanostructure deposition technology is a key problem in the emerging field of bottom-up nanotechnology of these bioinspired nanomaterials. It has been mainly performed by solution deposition or by a vapor transport method. In this study, we describe a new technique of physical vapor deposition (PVD) of biomolecules compatible with microelectronic technology allowing fabrication of dense and homogeneous peptide nanostructural materials. We present a deep insight into the self-assembly mechanism of peptide nanostructures deposited by our developed PVD technique, a study of their elementary growth stages by investigation of fine morphological and packing structure, molecular composition, and their chemical and physical properties. These have enabled us to develop a controllable deposition technology for fabricating peptide nanostructures.

Polymer Journal (2013) 45, 494-503; doi:10.1038/pj.2013.19; published online 20 March 2013

Keywords: diphenylalanine (FF); peptide nano structures; physical vapor deposition (PVD); self-assembly mechanism

\section{INTRODUCTION}

Experimental techniques for deposition of peptide nanomaterials A number of deposition methods for peptide nanostructures have been suggested. Most of them are based on solution techniques, and some are based on evaporation of the peptides and their deposition on a substrate. Reches and Gazit ${ }^{1}$ performed and studied solutiondeposition of the diphenylalanine, FF dipeptide, by dissolving the lyophilized FF-monomers in a highly volatile organic solvent to form monodispersed building blocks. The solvent then rapidly evaporates, forming a thin layer of vertically aligned peptide nanotubes (PNTs). Another solution-based approach was reported by Ryu and Park, ${ }^{2}$ who deposited a FF-highly volatile organic solvent solution onto a $\mathrm{Si}$ substrate under strictly anhydrous conditions, preventing waterassisted film growth, followed by aging the amorphous film in aniline vapor at temperatures above $100{ }^{\circ} \mathrm{C}$ to form vertically aligned crystalline peptide nanowires.

Semiconducting, single crystalline, peptide-based nanowires were synthesized through a vapor-transport process, ${ }^{3}$ using linear-FF (LFF) peptides as a precursor. These peptides were vaporized at $250{ }^{\circ} \mathrm{C}$ in an anhydrous argon atmosphere and transported downstream in a horizontal tube onto a $\mathrm{Si}$ substrate. As the peptides are heated to $250^{\circ} \mathrm{C}$, the linear peptides were converted into cyclo-FF (C-FF) peptides, formed horizontal nanowires on the substrate, with no apparent order or alignment.
A physical vapor deposition (PVD) method for forming vertically aligned peptide nanotubes was proposed by our group. ${ }^{4}$ Scanning electron microscope (SEM) data of the deposited PNTs revealed normally oriented, homogeneously distributed arrays with a density of $4 \times 10^{8}$ nanotubes $\mathrm{cm}^{-2}$. In this paper, we describe detailed studies of PVD technique, which was mainly applied to FF-peptides. We consider the self-assembly mechanism of the deposited FF-peptide nanostructures, study of their elementary growth stages by investigation of fine morphological and packing structure, molecular composition, and their chemical and physical properties. These have enabled us to develop a controllable deposition technology for fabricating peptide nanostructures.

PVD of peptide nanostructures

Although PVD is a simple, well-developed technique for inorganic nano-assemblies, when used on peptidic biomolecules, it becomes a more complex and unknown process due to the use of high evaporation temperature of biomolecules and vacuum conditions, which can strongly influence the self-assembly process ${ }^{4}$ as this is not the natural environment for such precursor materials. Therefore, in order to understand the process and to achieve the desired final nanostructures formed from these organic building blocks, we will follow the PVD self-assembly process and elucidate its various stages.

${ }^{1}$ School of Electrical Engineering, Iby and Aladar Fleischman Faculty of Engineering, Tel Aviv University Tel Aviv, Israel and ${ }^{2}$ Wolfson Applied Materials Research Center, Tel Aviv University, Tel Aviv, Israel

Correspondence: Professor G Rosenman, School of Electrical Engineering, Iby and Aladar Fleischman Faculty of Engineering, Tel Aviv University, Tel Aviv 69978, Israel. E-mail: gilr@eng.tau.ac.il

Received 10 December 2012; revised 2 January 2013; accepted 22 January 2013; published online 20 March 2013 
We show that PVD deposition process of FF-dipeptide self-assembly consists of a few elementary stages, in which the L-FF biomolecules first adhere to the substrate and form a thin amorphous layer, which grows into nanoclusters. We demonstrate that at the next stage the self-assembly could proceed in two ways:

(a) Open-ended nanotubes composed from L-FF molecules when small concentrations of water molecules exist within the vacuum chamber (low vacuum).

(b) Closed-ended nanofibers, which consist of C-FF molecules, caused due to a molecular transformation (cyclization process) of the FF molecule at high temperatures of deposition.

\section{EXPERIMENTAL PROCEDURE}

\section{PVD technique for peptide nanostructures}

The experimental set up of the PVD system, developed by our group for the assembly of bioinspired nano-structures is presented is this section.

A schematic diagram of our PVD system designed to deposit biomolecules by thermal evaporation is shown in Figure 1. The deposition procedure was conducted in the vacuum chamber ${ }^{1}$ which contains several sub-systems: a vacuum control system, ${ }^{2}$ a thickness control system, ${ }^{3}$ a turbomolecular pump, ${ }^{4}$ a vacuum gauge, ${ }^{5}$ and a heating control system for the heater ${ }^{6}$ and substrate ${ }^{7}$ holders. The vacuum pressure (up to $\sim 2 \times 10^{-6}$ mbar) is provided by a vacuum turbomolecular pump system (Pfeiffer, Asslar, Germany). The heating system contains two identical individual resistance heaters in which the temperature is controlled and stabilized independently. Each system includes four individual heating units for the substrate holders and four evaporators in the form of copper boats. The substrate holders ${ }^{9}$ are located above the precursor material holders ${ }^{10}$. The distance between the substrate and the powder holders may be gradually varied in the range of $2-5 \mathrm{~cm}$. The heater of the substrate

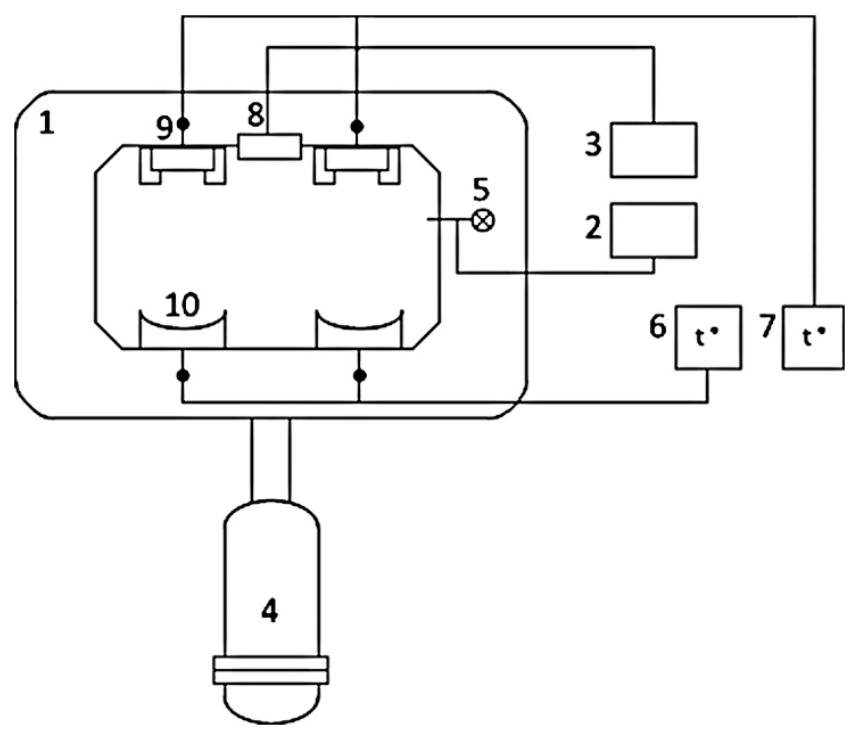

Figure 1 Physical vapor deposition set up used for deposition of biomolecular nano-structures: 1 , vacuum chamber; 2 , vacuum control system; 3, thickness control system; 4, vacuum turbo-molecular pump system; 5 , vacuum gauge; 6 , heating control system of substrate holder; 7 , heating control system of the biomaterial precursor; 8 , piezoelectric quartz for thickness control; 9, substrate holder; 10, raw biomaterial holder. holder is controlled and stabilized in a temperature range between $T_{\mathrm{S}} \sim 25-150^{\circ} \mathrm{C}$. The evaporator's heater is used for sublimation of the peptide precursor from the holder in the temperature range of $T_{\mathrm{H}} \sim 25-300^{\circ} \mathrm{C}$. Thickness monitoring of the evaporated biomaterial is achieved by the use of a piezoelectric quartz unit. ${ }^{8}$

In this study, linear and cyclic FF precursor powders were deposited onto $\mathrm{SiO}_{2}$ substrates. The substrates were washed and sonicated in successive baths of acetone and isopropanol. Approximately $4.5 \mathrm{mg}$ of the peptide precursor was used as the source material. The material was sublimed at a pressure of $10^{-6} \mathrm{mbar}$, the substrate temperature $\left(T_{\mathrm{S}}\right)$ was set to $\sim 90^{\circ} \mathrm{C}$, and the heater temperature $\left(T_{\mathrm{H}}\right)$ was set to various temperatures and deposition times, according to the objective of the experiment. The samples were kept in a desiccator cabinet upon removal from the PVD system.

\section{Materials and methods}

The molecules deposited in this work are diphenylalanine (FF) and C-FF lyophilized powder purchased from Bachem (Bubendorf, Switzerland)

Atomic force microscopy. Topography features were observed by atomic force microscopy Dimension 3100 (Veeco Instruments Inc., Santa Barbara, CA, USA) in Tapping mode. Ultrasharp Si-tips with a 2 -nm radius of curvature were used.

Environmental scanning electron microscope. In the present work, environmental scanning electron microscope (ESEM) was utilized both in its dry mode for and in its wet mode to follow the dynamic process of PNT formation after the first PVD. The ESEM used was a Quanta 200 FEG model (The Wolfson Applied Materials Research Center, Tel Aviv University).

Dynamic wetting studies were done in wet mode, using a Peltier stage. The sample was put on the stage, which was stabilized at $2{ }^{\circ} \mathrm{C}$, while vapor pressure was maintained at 5 Torr to avoid condensation. The pressure was then slowly increased, leading to controlled water condensation on the sample. The final pressure was 6 Torr, allowing stable imaging conditions.

$X$-ray diffraction. X-ray diffraction data were collected with $\mathrm{CuK} \alpha$ radiation on $\Theta-\Theta$ Powder Diffractometer Scintag X1 (Scintag Inc., Sunnyvale, CA, USA) equipped with a liquid nitrogen cooled Ge solid-state detector.

Time-of-flight secondary ion mass spectrometer. Time-of-flight (ToF)-secondary ion mass spectrometer (SIMS) analysis was applied in order to determine chemical surface compositions. ToF-SIMS measurements were carried out using a Physical Electronics TRIFT II ToF-SIMS instrument using a $15-\mathrm{kV} \mathrm{Ga}+$ primary ion gun (The Wolfson Applied Materials Research Center, Tel Aviv University).

Electrospray mass spectrometry. Electrospray mass spectrometry was carried out in order to determine chemical composition of the bulk of the PVD films. PVD films were immersed in 2-propanol and sonicated for $5 \mathrm{~min}$ for film removal and dissolution. Mass spectrometry analysis was performed using SYNAPT High Definition Mass spectrometer (waters corporation, Milford, MA, USA) with ESI source in positive and negative modes with a mixture of water and methanol. 


\section{RESULTS}

Self-assembly stages of PVD-deposited peptide nanostructures

The deposition process of the sublimed peptides at vacuum pressure of $10^{-6} \mathrm{mbar}$ was examined versus heater temperature increase from room temperature up to $T_{\mathrm{H}} \sim 300^{\circ} \mathrm{C}$, using a piezoelectric quartz crystal microbalance (QCM) method.

Two major peaks in the QCM deposition-rate signal were observed as the heater temperature increased over time, as displayed in Figure 2. It can be seen that there are two major peaks in deposition rate, the first peak is observed between $T_{\mathrm{H}} \sim 140-190{ }^{\circ} \mathrm{C}$, and the second appears between $T_{\mathrm{H}} \sim 220-300{ }^{\circ} \mathrm{C}$, implying two stages of peptide sublimation and deposition onto the substrate.

The first stage of PVD: initial amorphous linear FF film

The first stage of the process detected by the QCM sensor begins at $140{ }^{\circ} \mathrm{C}$. Accordingly, $T_{\mathrm{H}}$ was set to $140{ }^{\circ} \mathrm{C}$ for $\sim 20 \mathrm{~min}$, to obtain a layer with a detectable amount of deposited molecules on a $\mathrm{SiO}_{2}$ substrate. Atomic force microscopy measurements of the deposited film show a thin layer of elongated peptide nano-islands of $\sim 15.7 \mathrm{~nm}$ height (Figure 3a) and 50-500 nm width (Figure 3b).

ToF-SIMS analysis was carried out on the precursor powder, as purchased, as well as on the peptide nano islands. Figure 4 shows the characteristic $313 \mathrm{~m} / \mathrm{z}$ peak of the positive ion spectrum of L-FF Figure $4 \mathrm{a}^{5}$ The nano-islands formed at $T_{\mathrm{H}} \sim 150{ }^{\circ} \mathrm{C}$ show a major $313 \mathrm{~m} / \mathrm{z}$ peak Figure $4 \mathrm{~b}$, indicating the adsorption of the monomers

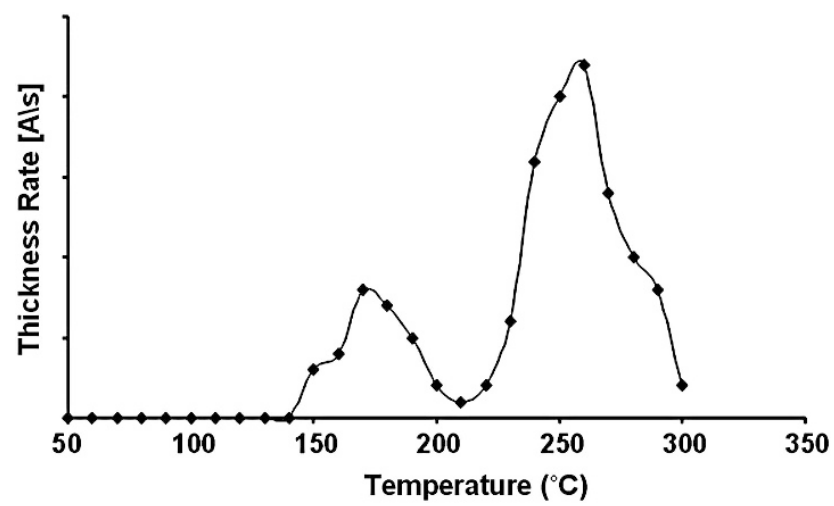

Figure 2 Deposition rate as a function of heater temperature $\left(T_{H}\right)$, vacuum pressure of $10^{-6}$ mbar. Two major peaks are seen. onto the substrate. Further deposition of FF peptides for $\sim 20 \mathrm{~min}$ at $T_{\mathrm{H}} \sim 190^{\circ} \mathrm{C}$, which is the upper limit of the first deposition peak according to the QCM, shows a dense layer of amorphous aggregates, as seen in the SEM cross-section in Figure 5. The ToF-SIMS positive ion spectrum of this film (Figure 6) shows a major peak at $313 \mathrm{~m} / \mathrm{z}$, corresponding to the L-FF and a minor $295 \mathrm{~m} / \mathrm{z}$ peak. It should be emphasized that extension of deposition time for over $1 \mathrm{~h}$ at this temperature did not form any changes in the surface, no nanotubular structures were observed.

The second PVD stage: FF-Peptide Nano Fibers

When the PVD process is extended and higher heater temperatures $\left(T_{\mathrm{H}} \sim 225-300^{\circ} \mathrm{C}\right)$ are reached, a second peak appears on the QCM,
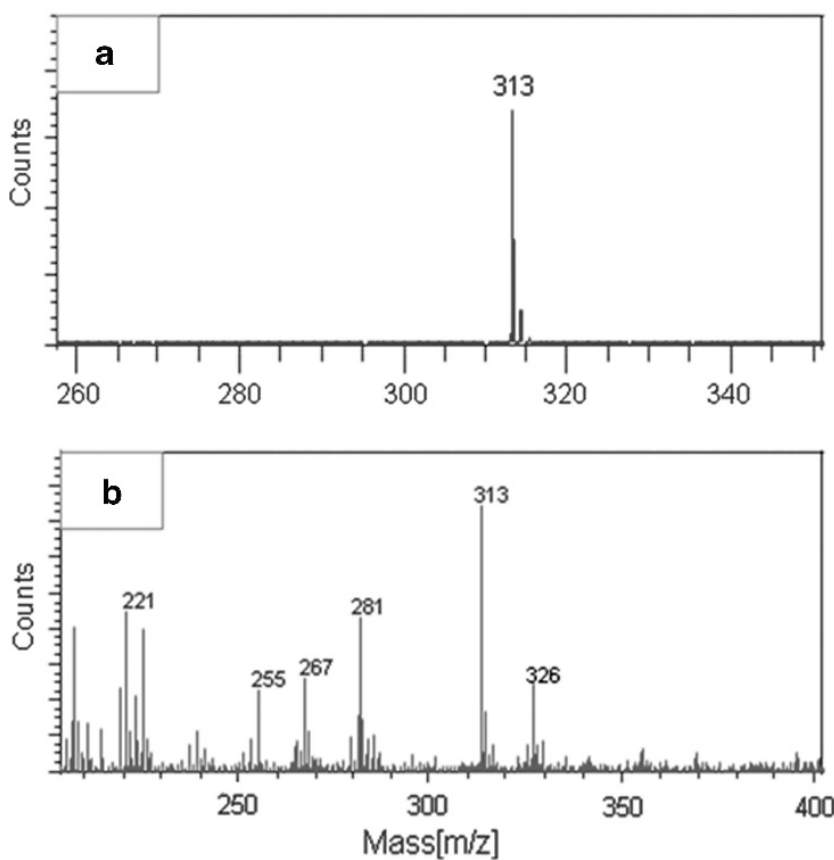

Figure 4 Time-of-flight-secondary ion mass spectrometer spectra of (a) FF monomer precursor powder, (b) physical vapor deposition peptide nano-islands, $T_{\mathrm{H}}<150^{\circ} \mathrm{C}$, only linear- $\mathrm{FF}(313 \mathrm{~m} / \mathrm{z})$ reached the substrate. A full color version of this figure is available at Polymer Journal online.
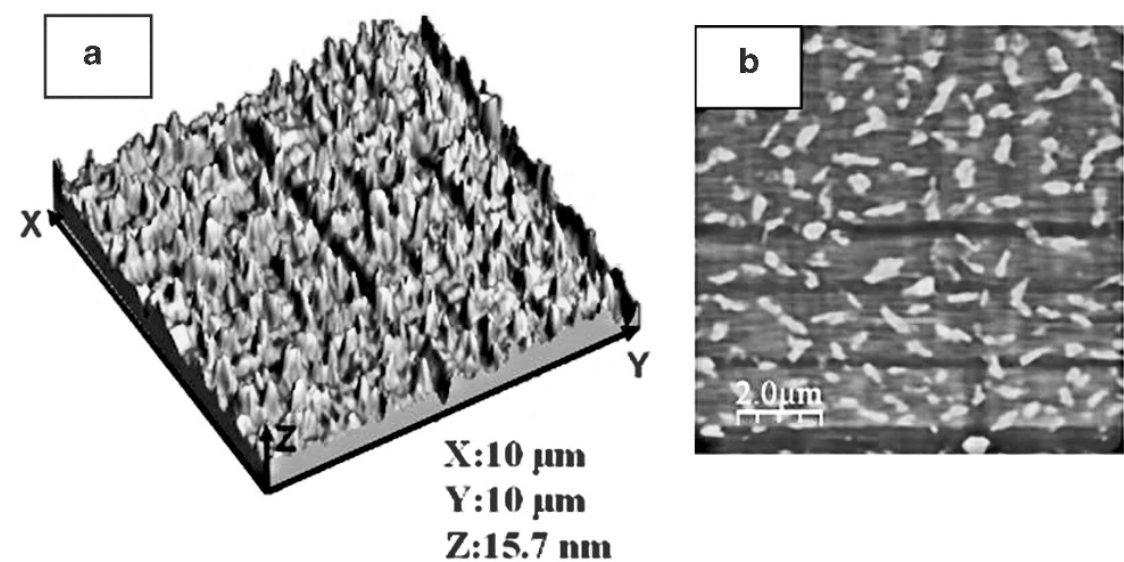

Figure 3 Atomic force microscopy measurements of first peptide layer, vacuum pressure of $10^{-6} \mathrm{mbar}$. (a) Topographic view, (b) Two-dimensional view of peptide nano-islands deposited on a $\mathrm{SiO}_{2}$ substrate, $T_{\mathrm{S}} \sim 90^{\circ} \mathrm{C}, T_{\mathrm{H}} \sim 140^{\circ} \mathrm{C}, t=20 \mathrm{~min}$. A full color version of this figure is available at Polymer Journal online. 
indicating an additional sublimation-deposition stage, and the formation of a new layer on the substrate.

In order to view the preliminary growth of the second deposition stage, the heater temperature was increased slowly, by $10^{\circ} \mathrm{C}$ every $5 \mathrm{~min}$, until the second QCM signal appeared at $T_{\mathrm{H}} \sim 225^{\circ} \mathrm{C}$.

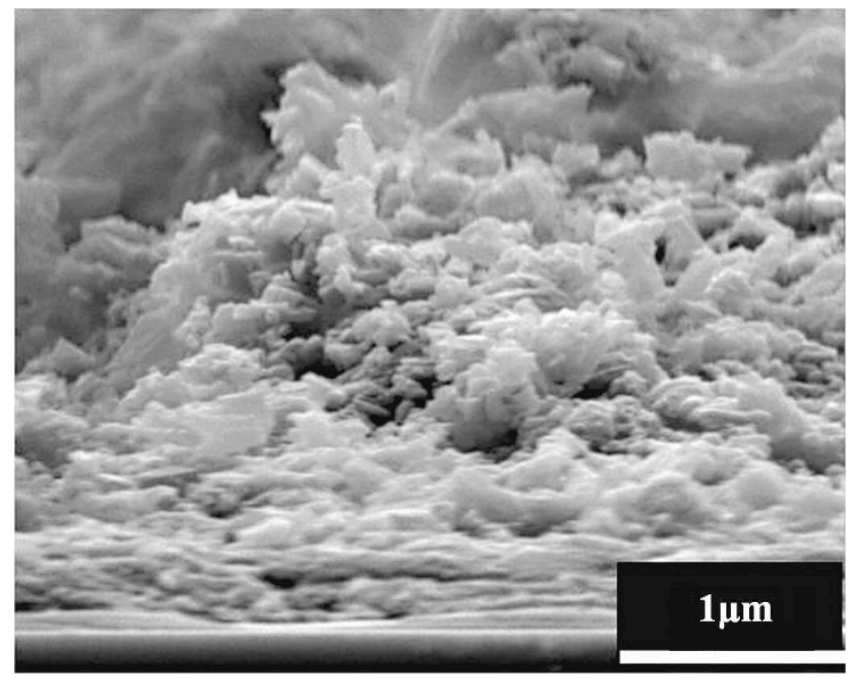

Figure 5 Scanning electron microscope cross-section of an FF physical vapor deposition film deposited for $20 \mathrm{~min}$ at $T_{\mathrm{H}} \sim 190^{\circ} \mathrm{C}$, vacuum pressure of $10^{-6} \mathrm{mbar}$.

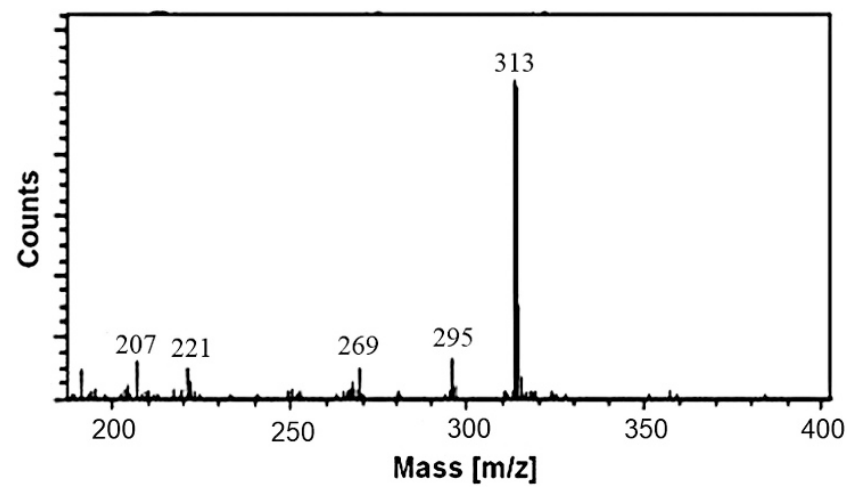

Figure 6 Time-of-flight-secondary ion mass spectrometer-positive ion spectrum of a PVD film formed at $T_{\mathrm{H}} \sim 190^{\circ} \mathrm{C}$. Major $313 \mathrm{~m} / \mathrm{z}$ peak, minor $295 \mathrm{~m} / \mathrm{z}$.
When the signal was detected, the heater was shut down, in order to minimize additional deposition. Owing to a slow heater temperature decrease $\left(\sim 1^{\circ} \mathrm{C} \min ^{-1}\right)$, additional deposition occurred after turning off the heater. Figure $7 \mathrm{a}$ shows a cross-section of a film formed at a slow deposition rate, where a thick $(\sim 5 \mu \mathrm{m})$ amorphous layer appeared, and the beginning of the growth of the second closedended peptide nanofiber layer is seen in the horizon (white arrow) and in the top view in Figure $7 \mathrm{~b}$.

When changing the deposition method by rapidly increasing $T_{\mathrm{H}}$ $\left(\sim 8{ }^{\circ} \mathrm{C} \mathrm{min}^{-1}\right)$ and leaving the heater at the second deposition temperature of $T_{\mathrm{H}} \sim 225^{\circ} \mathrm{C}$ for an extended time, the thickness of the different layers was altered. Figure 8 shows a top view and crosssections of a film grown at $T_{\mathrm{H}} \sim 225^{\circ} \mathrm{C}$ for $4 \mathrm{~h}$. It can be seen that the two layers still exist in the film, but their thicknesses are now different. Closed-ended fibers are seen in the top view (Figure 8a), and the cross-section (Figure 8b) shows the two layers composing the film: the top layer of vertically aligned peptide nano fibers (PNFs) extend to $\sim 1.3 \mu \mathrm{m}$ (black arrow), which are located on top of an amorphous layer (white arrow), which is $\sim 500 \mathrm{~nm}$ thick. Figure $8 \mathrm{c}$ shows the amorphous aggregates of the bottom layer, with a width in the range of $100-500 \mathrm{~nm}$.

Depositing the FF peptides by setting the heater temperature to $T_{\mathrm{H}} \sim 250{ }^{\circ} \mathrm{C}$, the amorphous layer became less apparent, and the PNFs became the dominant portion of the film. The films and the precursor powder that remained in the precursor holder after a PVD session of the second stage $\left(T_{\mathrm{H}} \sim 250{ }^{\circ} \mathrm{C}\right)$, were collected and analyzed for their chemical composition using ToF-SIMS, as shown in Figure 9. The positive ion spectrum (Figure $9 \mathrm{a}$ ) displays only a $295 \mathrm{~m} / \mathrm{z}$ signal. As the pulsed ion beam of the ToF-SIMS collects molecules only from the outermost surface of the sample, thus showing that the outermost layer of the film carries a signal of $295 \mathrm{~m} / \mathrm{z}$. Figure $9 \mathrm{~b}$ shows that the powder that did not sublime after the PVD session still comprises some L-FF $(313 \mathrm{~m} / \mathrm{z})$ in addition to a prominent $295 \mathrm{~m} / \mathrm{z}$ signal.

Extending the deposition to a full PVD process by setting the heater to $T_{\mathrm{H}} \sim 250^{\circ} \mathrm{C}$ for $35 \mathrm{~min}$, followed by an increase to $T_{\mathrm{H}} \sim 300^{\circ} \mathrm{C}$ for $20 \mathrm{~min}$, a $\sim 6 \mu \mathrm{m}$ thick, dense layer of vertically aligned, closed-ended PNF film is formed, and the amorphous layer is not observed (Figure 10).

Chemical composition of the top layer and of the bulk of the PNF film was analyzed through ToF-SIMS and electrospray mass spectrometry-mass spectrometry (Figure $11 \mathrm{a}$ and $\mathrm{b}$, respectively).

It can be seen that the analysis of the outermost part of the PVD FF-PNFs film shows only a $295 \mathrm{~m} / \mathrm{z}$ peak (the 120 and $91 \mathrm{~m} / \mathrm{z}$ molecular weights correspond to hydrocarbon compounds of $\mathrm{C}_{7} \mathrm{H}_{7}$
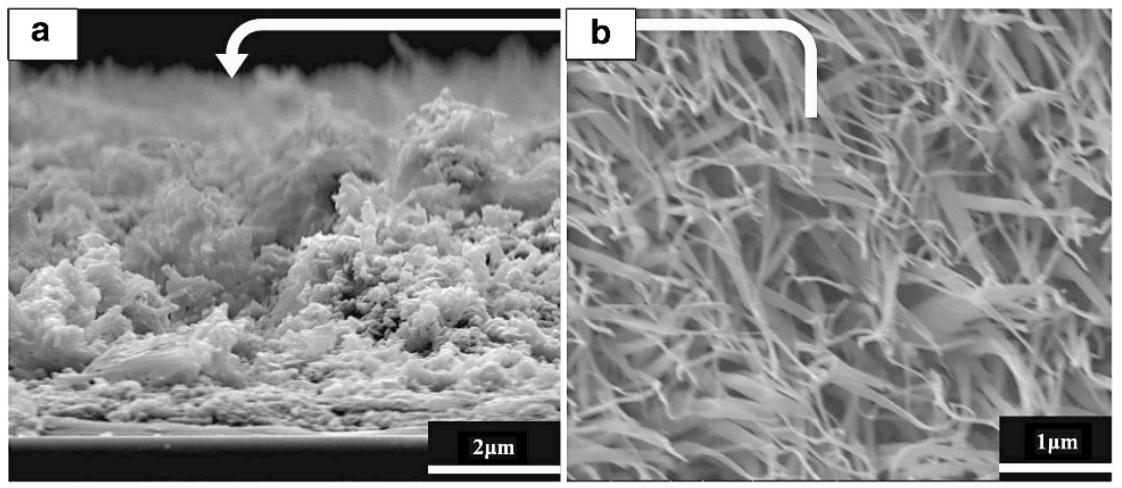

Figure 7 Scanning electron microscope images of preliminary growth of the second physical vapor deposition stage $\left(T_{H} \sim 225^{\circ} \mathrm{C}\right)$, vacuum pressure of $10^{-6}$ mbar. (a) Cross-section of amorphous bottom layer, peptide nano fibers seen in the horizon, (b) top view, closed-ended PNF. 


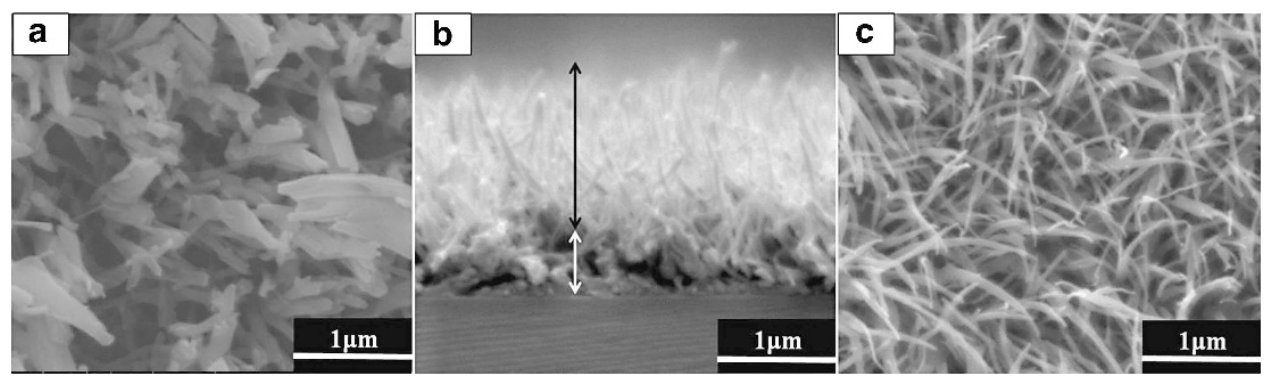

Figure 8 Scanning electron microscope image of physical vapor deposition film of FF monomer deposited for $4 \mathrm{~h}$ at $T_{\mathrm{H}} \sim 225^{\circ} \mathrm{C}$, vacuum pressure of $10^{-6}$ mbar. (a) Amorphous aggregates of the bottom layer (b) cross-section, white arrow shows the amorphous layer, black arrow shows peptide nano fiber (PNF) layer (c) top view, closed-ended PNF.
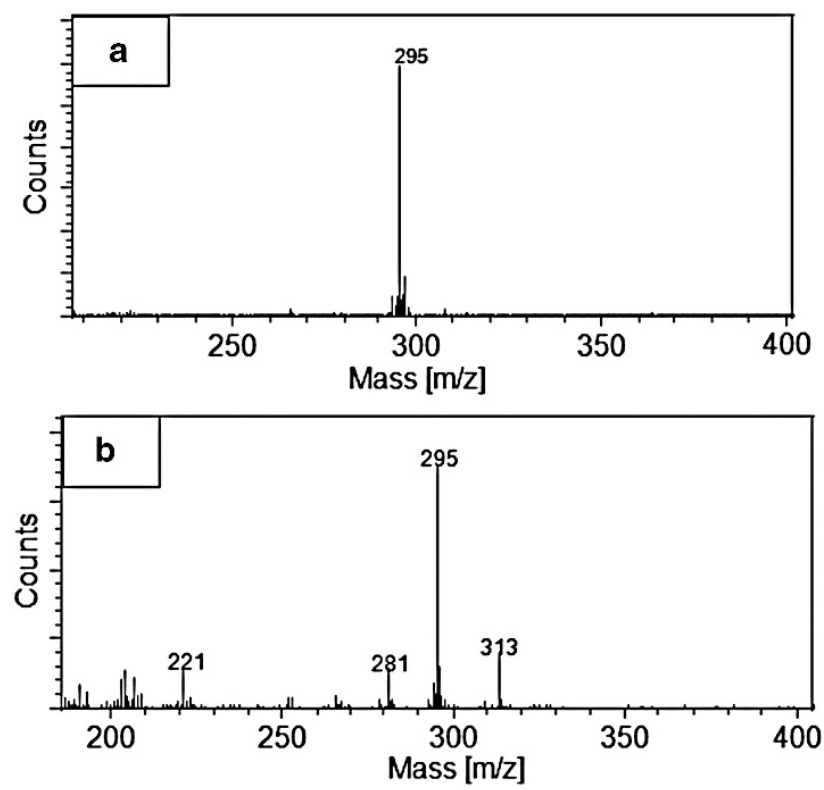

Figure 9 Time-of-flight-secondary ion mass spectrometer spectra of second physical vapor deposition stage, $T_{\mathrm{H}} \sim 250^{\circ} \mathrm{C}$. (a) Deposited film, cyclo-FF $(295 \mathrm{~m} / \mathrm{z})$ signal. (b) Remaining powder in heater shows a major $295 \mathrm{~m} / \mathrm{z}$ and a small $313 \mathrm{~m} / \mathrm{z}$ peak.

and $\mathrm{C}_{8} \mathrm{H}_{10} \mathrm{~N}$, respectively, which are structures that are commonly attributed to varying degrees of fragmentation of phenylalanine, specifically fragments of, or containing, the aromatic ring sidechain $\left.^{6}\right)$. However, the electrospray mass spectrometry-mass spectrometry negative ion spectrum of the same film shows that both 311 and $293 \mathrm{~m} / \mathrm{z}$ peaks are abundant in the bulk of the film, indicating that linear diphenylalanine still exists in the film, though not in its top layer.

The crystal structure of the peptide films formed at different stages of the PVD process were analyzed by X-ray diffraction. Figure 12 follows the crystallographic changes that the film undergoes at heater temperatures of 190,225 and $300^{\circ} \mathrm{C}$ (light gray, dark gray and black, respectively). It can be seen that the first deposition stage leads to the formation of an amorphous film, indicated by a wide halo and showing no diffraction peaks in the X-ray diffraction spectrum. Increasing the deposition temperature to $225^{\circ} \mathrm{C}$, the amorphous layer remains, but the beginning of a nucleation of a crystal structure, exhibited by the (002), (102), (113) and (023) Miller indices, is apparent. Extending the process and increasing the heater

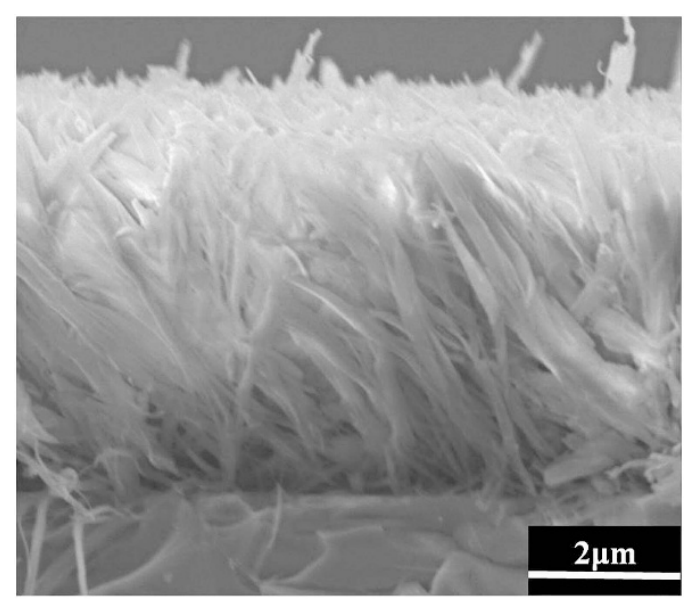

Figure 10 Scanning electron microscope cross-section of a peptide nano fiber film formed at $T_{\mathrm{H}} \sim 300^{\circ} \mathrm{C}$, vacuum pressure of $10^{-6} \mathrm{mbar}$.
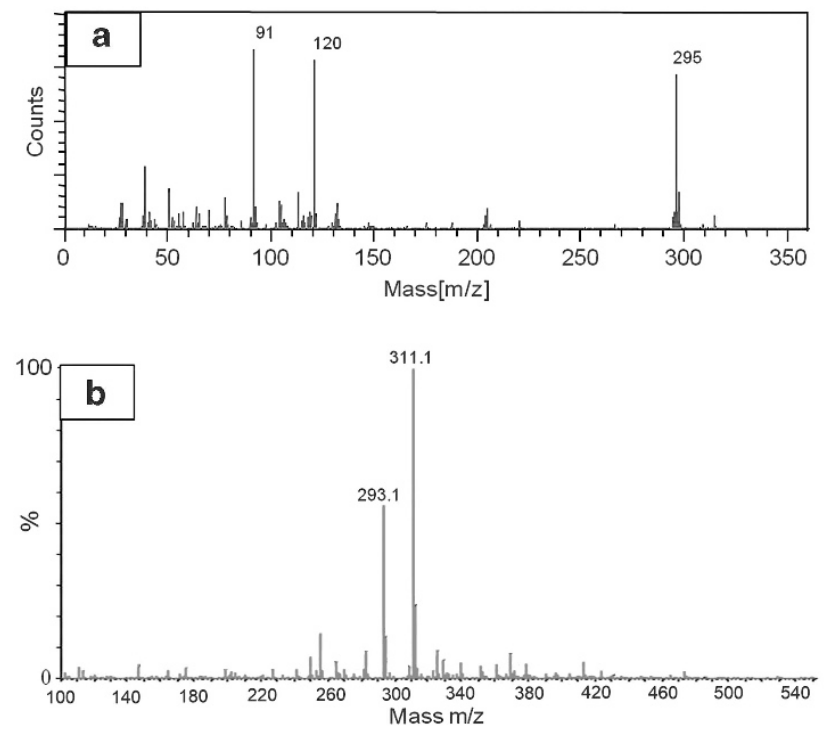

Figure 11 Full physical vapor deposition peptide nano fiber film chemical analysis. (a) Time-of-flight-secondary ion mass spectrometer-positive ion spectrum, $295 \mathrm{~m} / \mathrm{z}$ peak, (b) electrospray mass spectrometry-mass spectrometry-negative ion spectrum of the dissolved film shows both 311 and $293 \mathrm{~m} / \mathrm{z}$ peaks. A full color version of this figure is available at Polymer Journal online. 
temperature to $300{ }^{\circ} \mathrm{C}$ leads to a more defined crystal structure, and as the black line in Figure 12 demonstrates, the amorphous halo widens and moves to the right, indicating that the amorphous layer has become less dominant and the crystal structure has grown.

The crystal symmetry or the PVD FF-PNFs film grown at $T_{\mathrm{H}} \sim 300^{\circ} \mathrm{C}$ shows the exact same pattern as that of thermally

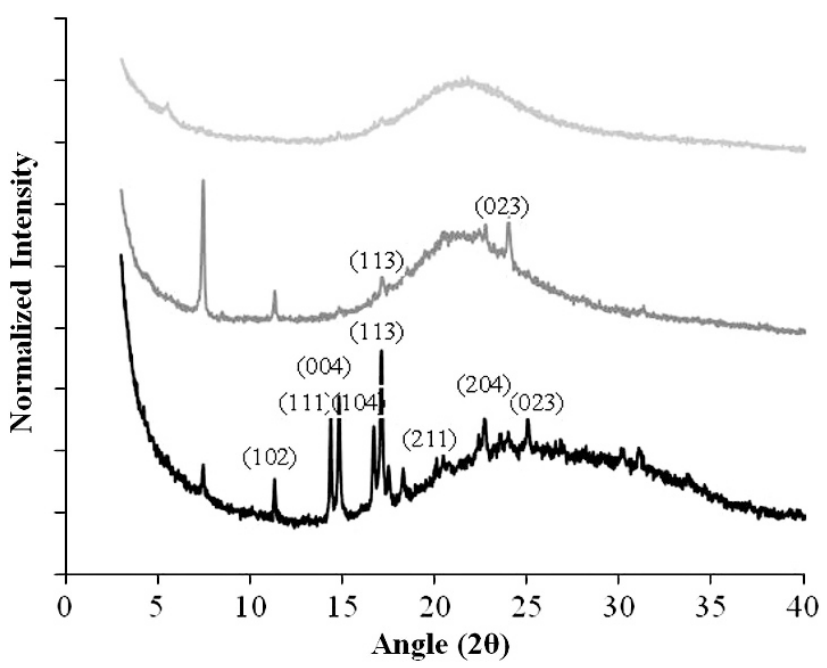

Figure 12 X-ray diffraction spectra of physical vapor deposition (PVD) PN films deposited at $T_{\mathrm{H}} \sim 190^{\circ} \mathrm{C}$ (light gray), $225^{\circ} \mathrm{C}$ (dark gray) and full PVD peptide nano fiber film, $300^{\circ} \mathrm{C}$ (black).

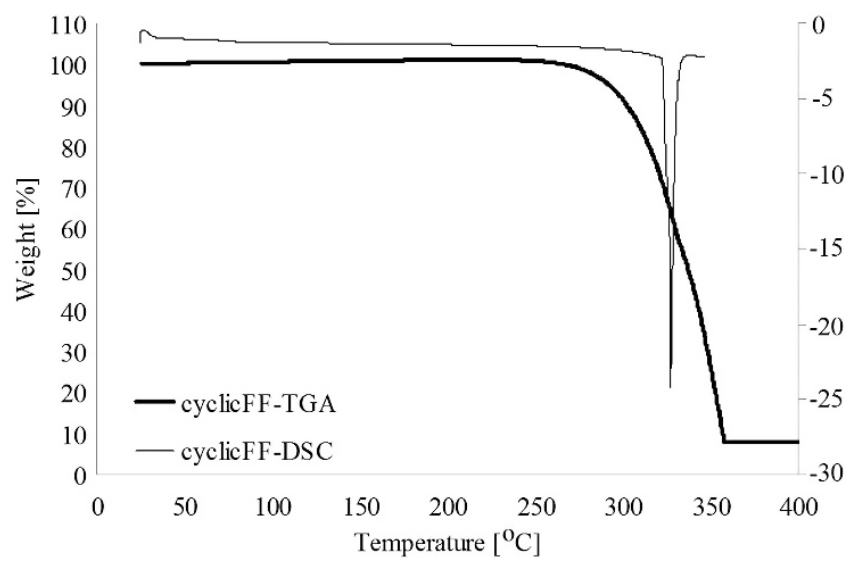

Figure 13 Thermogravimetric analysis differential scanning calorimetry of cyclo-diphenylalanine precursor powder.

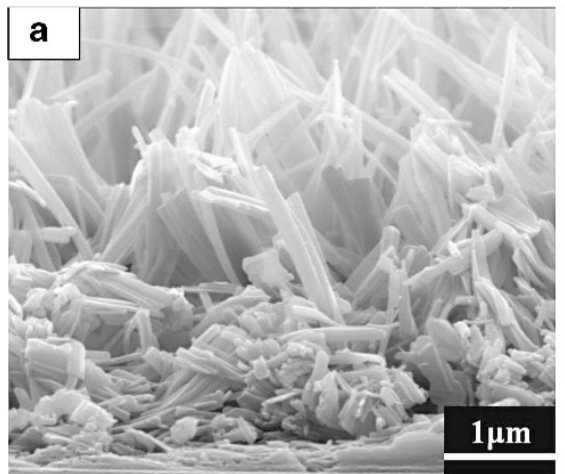

induced, solution-deposited FF-PNFs, which were investigated by our group and compared with native FF-PNTs. ${ }^{5}$ The Miller indices (hkl) assignments in Figure 12 of $T_{\mathrm{H}} \sim 300^{\circ} \mathrm{C}$, correspond to the same orthorhombic unit cell (Pbca: $a=10.31, b=8.42$ and $c=23.87 \AA$ ) as was found for the thermally treated FF-PNF.

This orthorhombic $D_{2 h}$ symmetry is related to a centrosymmetric space group structure. Studies undertaken with two different peptide nanotubular FF-PNT and FF-PNF showed that PNT possessing an asymmetric structure demonstrate piezoelectric effects ${ }^{7}$ and second harmonic generation ${ }^{7,8}$ while both effects disappeared in centrosymmetric FF-PNFs.

\section{Nano Fibers deposited from cyclo-diphenylalanine precursor}

Thermal analysis (Figure 13) of the diketopiperazine was executed, showing thermal stability up to $\sim 250^{\circ} \mathrm{C}$, with no additional weight loss as occurs in the L-FF. The weight loss of this monomer begins at the same temperature as that of the linear peptide after its cyclization reaction..$^{5}$

\section{PVD of cyclic diphenylalanine}

C-FF was deposited by PVD onto $\mathrm{SiO}_{2}$ substrates at $T_{\mathrm{H}} \sim 250{ }^{\circ} \mathrm{C}$, $T_{\mathrm{S}} \sim 85^{\circ} \mathrm{C}$ and a pressure of $10^{-6} \mathrm{mbar}$. The films formed under these conditions were characterized and compared with L-FF PVD films (Figures 7, 8 and 10), which were formed under similar conditions.

Figure 14 displays a cross-section and a top view of a PVD film formed using C-FF as a precursor. It can be seen that the PVD film, self-assembled from the Cyclic FF precursor, is composed of vertically aligned, close-ended peptide nanofibers. This morphology highly resembles that of the films deposited from L-FF precursor during the second PVD stage at high temperatures $\left(T_{\mathrm{H}} \sim 220-300^{\circ} \mathrm{C}\right)$ when FFmolecular transformation also leads to fabrication of PNF structure (Figures 7, 8 and 10).

The crystal structure of the C-FF PVD film was analyzed by X-ray diffraction, as displayed in Figure 15. High resemblance can be noticed between PVD films obtained by depositing L-FF as the precursor powder at high $T_{\mathrm{H}}$, and those obtained by using cyclic diphenylalanine as the precursor (black and gray, respectively). The inset in Figure 15 shows an X-ray diffractogram comparing between a L-FF PVD film and C-FF precursor powder. It can be seen that all three structures are highly similar, yet the peak at $37^{\circ}$, which is seen in the cyclic forms (PVD and powder), is not apparent in the L-FF PVD film.

\section{Open-ended peptide nanotubes}

Growth of open-ended PNTs in the ESEM chamber. We found that PVD linear FF amorphous film deposited at high vacuum $10^{-6} \mathrm{mbar}$

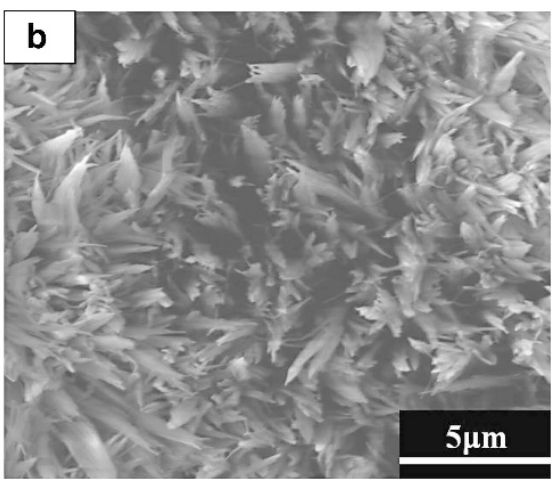

Figure 14 Scanning electron microscope image of a cyclo-FF physical vapor deposition film (a) cross-section, (b) top view. 


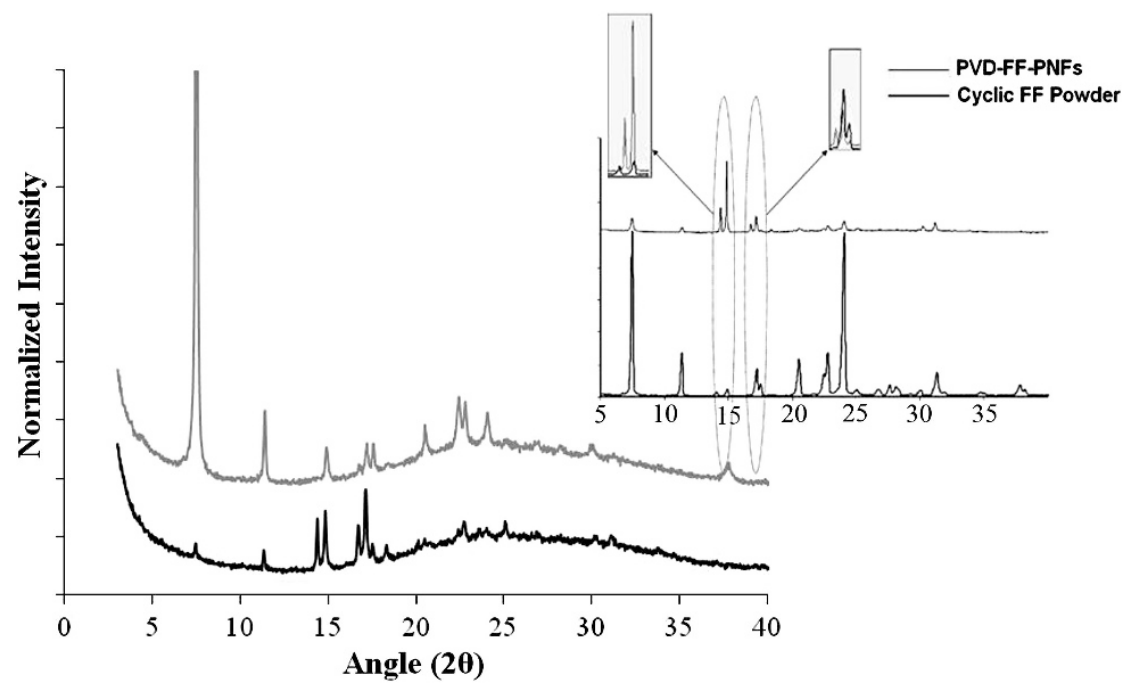

Figure 15 X-ray diffraction of physical vapor deposition (PVD) films from C-FF (top, gray) and from L-FF (bottom, black). Inset: cyclo-FF (C-FF) precursor powder (bottom, black), compared with linear-FF (L-FF) PVD film deposited at high temperature. PNF, peptide nano fibers.
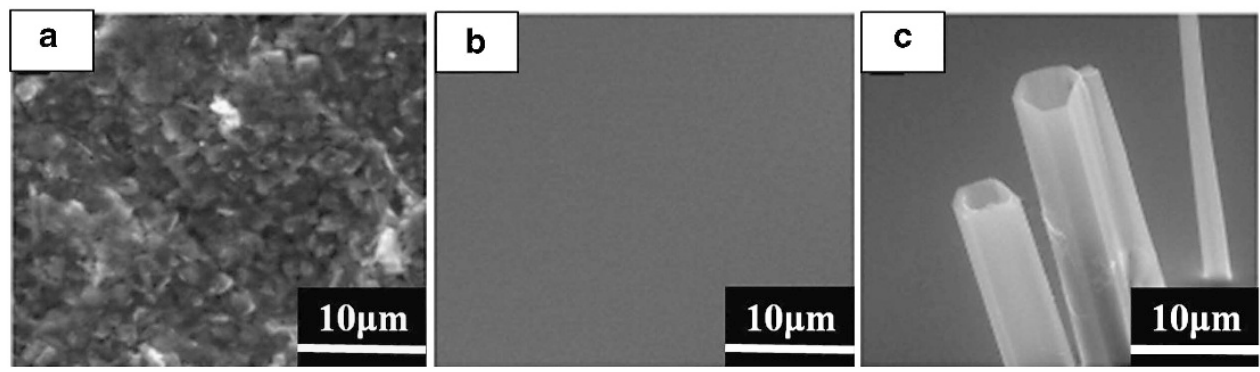

Figure 16 Environmental scanning electron microscope image of (a) amorphous peptide layer before wetting process, (b) amorphous peptide layer after full immersion in water and (c) FF-PNTs formed inside the ESEM chamber with water.

did not grow into nanotubular structure and extension of the time deposition leads to the growth of the FF-film only.

However, the same FF-film fabricated at low deposition temperature at vacuum pressure of $10^{-6} \mathrm{mbar}$ (Figure 5), was used for observation of a growth process of nanotubular structures in low vacuum (a few mbars) under high humidity conditions. These in-situ studies have been performed by using an ESEM. In this tool, the low vacuum pressure in the chamber allows to apply an unusual experimental environment in which the humidity in the chamber can be controlled. Such conditions allow water molecule condensation on the FF amorphous film surface. The ESEM experiments provide the ability to follow the self-assembling process of individual FF-PNT at the nanoscale upon changing the environment humidity and to understand the role of water molecules in the formation of openended FF-PNT. ${ }^{9-11}$

The ESEM studies began as the chamber was stabilized at $2{ }^{\circ} \mathrm{C}$ while the pressure was held at $5 \mathrm{mbar}$, in order to avoid vapor condensation. The peptide amorphous film is shown in Figure 16a. The ESEM pressure was raised at a rate of $\sim 0.1$ mbar every $15 \mathrm{~s}$, until full immersion of the amorphous FF-peptide layer in the water. The condensed water level was completely flat for a few seconds (Figure 16b), and a few seconds later a coordinated formation of highly ordered FF-PNTs was observed, as can be seen in Figure 16c.

Growth of open-ended FF-PNTs in a low-vacuum PVD system. Openended L-FF peptide nanotubes can also be assembled inside the PVD

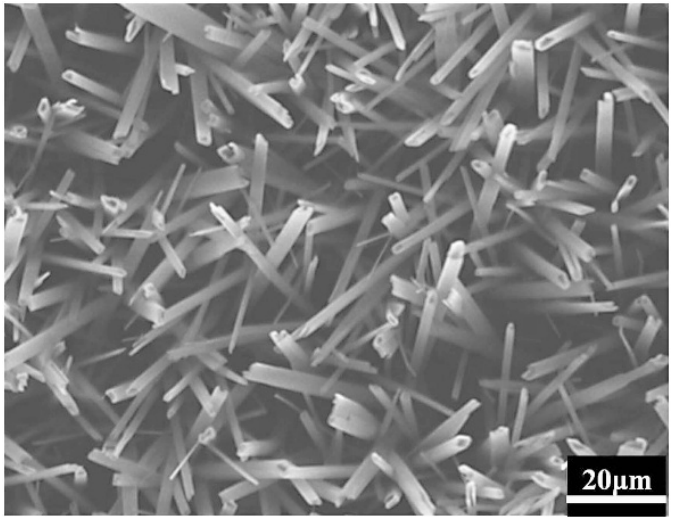

Figure 17 Scanning electron microscope image open-ended FF-PNTs by self-assembled in the physical vapor deposition system in low vacuum, $10^{-3} \mathrm{mbar}$.

system. The growth of a mixture of open-ended PNTs and closed-ended PNFs was done by setting the heater temperature to $T_{\mathrm{H}} \sim 240^{\circ} \mathrm{C}$, substrate temperature $T_{\mathrm{S}} \sim 85^{\circ} \mathrm{C}$ and the pressure in the PVD chamber to $10^{-3}$ mbar. Deposition procedure took $40 \mathrm{~min}$.

The deposited peptide layer was analyzed using SEM, as displayed in Figure 17. This figure distinctly shows the existence of a majority of open-ended PNTs having the same morphology as those observed by 


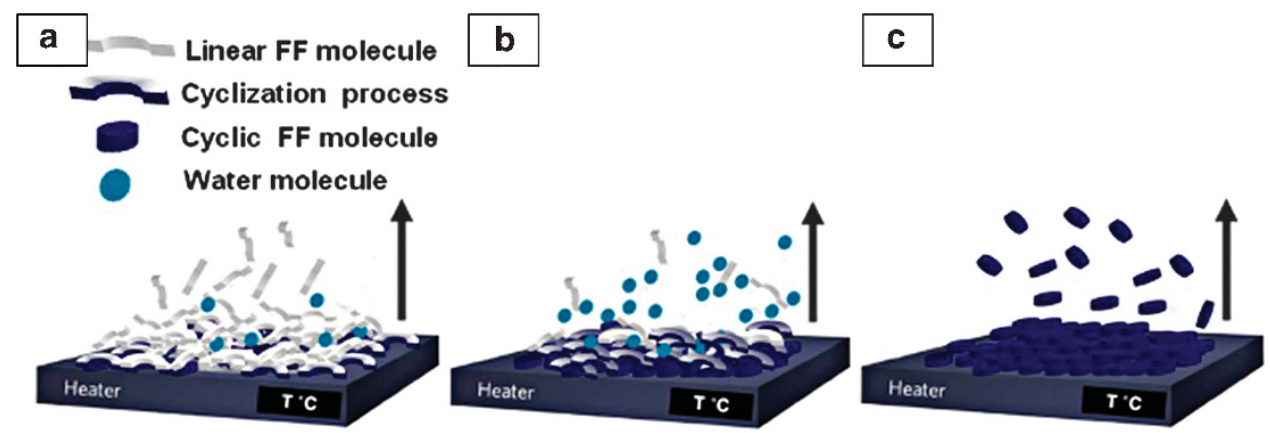

Figure $18 \mathrm{~A}$ possible model for the physical vapor deposition of linear-FF (L-FF). (a) Sublimation of L-FF molecules during heating to $T_{\mathrm{H}}<190^{\circ} \mathrm{C}$. (b) Release of water molecules during cyclization process and sublimation of a small amount of L-FF molecules while heating up to $T_{H} \sim 250{ }^{\circ} \mathrm{C}$. (c) Sublimation of remaining cyclo-FF molecules while heating up to $T_{\mathrm{H}} \sim 300^{\circ} \mathrm{C}$.

Görbitz $^{9-11}$ and Gazit $^{1}$ who formed and analyzed self-assembled FF-PNTs in aqueous solutions. ${ }^{3,12-14}$

These results are completely consistent with the model developed by Gorbitz ${ }^{9-11}$ for self-assembly mechanisms and structures of openend FF-PNT. According to the studies, the role of the solvent is critical in self-assembly processes. In the case of diphenylalanine, the water molecules are necessary in order to allow the formation of hydrogen bonds between them and the $\mathrm{C}$ - and $\mathrm{N}$ - termini of the peptides, and are the driving force for the self-assembly of diphenylalanine molecules into nanotubes. The aqueous environment tends to form intermolecular hydrogen bonds between the carbonyl oxygen and the amide hydrogen. The electrostatic interactions between the $\mathrm{N}$ - terminal $\left(-\mathrm{NH}_{3}+\right)$ and the $\mathrm{C}$ - terminal $\left(-\mathrm{COO}^{-}\right)$and the strong hydrogen bonds between them further promote aggregation of the peptide molecules in a head-to-tail fashion. ${ }^{15}$

We clearly exhibit that the presence of water molecules have an important role in the self-assembling process of FF: as shown before, the vacuum chamber, which is normally pumped for long enough time and set to high-vacuum values, does not allow for the assembly of L-FF into tubes, as residual gases are desorbed from the vacuum chamber.

On the other hand, it is a well-known fact that water vapor is the dominant residual gas during pumping, and once the chamber is exposed to air, the water vapor from the air instantly fills the system. ${ }^{16}$ Assuming room temperature and a pressure of $10^{-3} \mathrm{mbar}$, a density of about 10 molecules $\mathrm{cm}^{-3}$ ref. 16 , composed mostly of water vapor for an unbaked chamber that has recently been exposed to the atmosphere, ${ }^{16}$ fills the chamber. As a result, and according to the SEM image, these conditions in the PVD system allow for enough water molecules to assist the L-FF in the PNT self-assembly.

By following the stages of the PVD process of FF-monomer at high-vacuum conditions, which can be done in various temperatures and times, two different layers can be assembled: an amorphous layer and FF-PNF structure. The bottom layer fabricated at $T_{\mathrm{H}}<190^{\circ} \mathrm{C}$ is composed of linear FF-peptides, and has an amorphous morphology. FF-PNT cannot be fabricated under these conditions due to the lack of water molecules in the high vacuum. FF-PNF, which grow during the second stage of the PVD process at elevated temperatures $\left(T_{\mathrm{H}}>225^{\circ} \mathrm{C}\right)$, possess an orthorhombic $D_{2 h}$ symmetry. Open-ended FF-PNTs may be formed by two approaches: either by forming an amorphous film composed of L-FF-peptides in the PVD system and further exposing them to a hydrated environment, as shown in the ESEM experiment, leading to rapid self-assembly into vertically aligned open-ended FF-PNTs, or by performing PVD under low vacuum conditions, allowing sufficient water molecules for the self- assembly process to take place. Both processes resulted in the same morphology as that observed by Görbitz and Gazit for open-ended FF-PNTs. ${ }^{9-11,17}$

Following thermal analysis of L-FF, reported previously by our group $^{5}$ and supported by research done by Park on the vapor transport method, ${ }^{3}$ and after thorough analysis of the PVD films, we have concluded that the linear FF molecule undergoes a backbone cyclization upon heating the precursor above $150^{\circ} \mathrm{C}$, leading to formation of the Phe-Phe-diketopiperazine (cyclo-FF, C-FF), which is the composition of the top layer FF-PNFs.

\section{A suggested model for PVD of diphenylalanine}

In light of the data presented above, we suggest a model for the mechanism of FF-nanostructures formed by PVD. A scheme of the model is presented in Figure 18.

The gradual elevation of the heater temperature leads to two major stages in the deposition of the peptidic film. At first, as the temperature is raised up to $T_{\mathrm{H}}<190^{\circ} \mathrm{C}$, L-FF molecules, most likely from the top part of the powder, which are the least affected by the heater, yet are surrounded by a vacuum environment of $10^{-6} \mathrm{mbar}$, sublime and reach the substrate, forming a chemically homogenous, amorphous film. The continuous pumping of the system, in addition to high temperatures reached at this point do not allow water molecules to reach the substrate and interact with the peptides, therefore, no assembly of PNTs occurs at this stage. ${ }^{3}$

Analysis of the remaining precursor powder after deposition at $150{ }^{\circ} \mathrm{C}$ shows that the cyclization of the peptides begins to take place, as a new, minor $295 \mathrm{~m} / \mathrm{z}$ peak appears in the mass spectrum. The cyclized molecules are most likely to be those, which had closer contact to the precursor powder holder and have reached above $150^{\circ} \mathrm{C}$. Cyclization of the L-FF molecules occurs continuously upon raising the heater temperature up to $250^{\circ} \mathrm{C}$ and noncyclized peptides continue to sublime. At $T_{\mathrm{H}} \sim 225^{\circ} \mathrm{C}$, a second deposition stage begins, and nucleation of peptide nanofibers, composed of cyclized diphenylalanine occurs. Extending deposition durations of either the first or second stages, control over thicknesses of the amorphous or fibrous layers is gained. Increasing the heater temperature to $300{ }^{\circ} \mathrm{C}$, the remaining cyclic peptides sublime and deposit on the surface, self-assembling into vertically aligned FF-PNFs.

Performing the PVD in low-vacuum of $10^{-3}$ mbar allows for the assembly of open-ended FF-PNTs, as sufficient water molecules reside in the system and can interact with the L-FF that reaches the substrate. Films assembled at $T_{\mathrm{H}}<240{ }^{\circ} \mathrm{C}$ comprise a mixture of cyclic and L-FF, forming both FF-PNFs and FF-PNTs, respectively, to their components. 


\section{DISCUSSION}

Basic properties of peptide nanostructures and their future development and application

Many studies on peptide nanostructure's properties have shown fundamental physical properties, promising applications in a number of nanotechnological fields. The FF-PNTs are remarkably rigid structures, with a Young modulus of $19 \mathrm{GPa}$ and point stiffness of $160 \mathrm{~N} \mathrm{~m}^{-1} \cdot{ }^{18}$ Nanotubular structures can serve as structural elements for nanowires or nanoscaffolds, and as peptide nanotubes can be chemically modified, they can be specifically designed for biological systems. In a research done by Reches and Gazit ${ }^{17}$ regarding the structure of the FF-PNTs, they added ionic silver to the nanotubes in solution and found that silver nanoparticles were formed within the tube. Owing to this observation, they examined the ability of the nanotubes to serve as molds for casting metal nanowires, enabling formation of individual silver nanowires $\sim 20 \mathrm{~nm}$ in diameter.

FF-PNTs have demonstrated ferroelectric and related properties such as nonlinear optical and piezoelectric effects s,7,12,13 $^{5}$ based on their asymmetric nanocrystalline structure. Studies of optical properties allowed to find pronounced quantum confinement (QC) phenomena. $8,14,19,20$

The PNFs that were grown by PVD have shown an unusual step-like absorption spectrum, which implies the presence of a QC structure in the form of a quantum well. As QC structures produce remarkable changes in optical and electronic properties, photoluminescence can be observed in the visible blue region and thus allows to predict the potential development for a new generation of light emitting devices based on bio-inspired nanostructures. ${ }^{19,21}$

The PVD method for depositing FF-nanostructures was studied for surface modification, possibly serving various applications, such as ultracapacitors, highly hydrophobic (lotus-like) surfaces, PNT-based nanofluidic chips ${ }^{4}$ and light emitting materials. ${ }^{22}$ Electrochemical characterization of PNT-modified electrodes showed a 30-fold increase of the electrode double-layer capacitance density. The modified electrodes showed high chemical stability and good adhesion even after over 10000 continuous charge/discharge cycles in the electrolyte solution. The ordered nanoarrays were also studied for their ability to modify physical properties of various surfaces. The modification enabled the formation of superhydrophobic surfaces, owing to the formation of nanofibers with a high aspect ratio, resembling a 'lotus-type' array. An eightfold increase in hydrophobicity of a glass surface was achieved by depositing normally oriented PNF on the glass surface, increasing the contact angle of a water droplet from $\theta_{\text {nonmodified glass }} \sim 15^{\circ}$ to $\theta_{\text {PNT-modified glass }} \sim 125^{\circ}$. Higher contact angles were achieved by increasing the length of the precursor peptide, up to pentaphenyalalanine. Patterning of $\mathrm{SiO}_{2}$ surfaces with FF-PNTs was done by depositing the FF peptides through a shadowed mask. This technique allows the construction of FF-PNT-based microfluidic systems, as there is a wettability contrast between the hydrophilic silicone dioxide and the hydrophobic PNTs.

FF-PNTs have shown to undergo an irreversible structural phase transition upon heating above $150{ }^{\circ} \mathrm{C}$, leading to profound variation in their nano- and microscopic properties. ${ }^{5}$ The solution-assembled FF-PNT is formed from L-FF dipeptides, and carries properties such as a crystallographic sixfold hexagonal symmetry with a tubular structure containing multiple hydrophilic channels, physical properties such as strong shear piezoelectric activity, exceptional QC and hydrophilicity. The thermally treated FF-PNTs undergo a molecular transformation to their cyclic form and exhibit a morphological transition to a peptide nanofiber (FF-PNF), with an orthorhombic unit cell, which is centrosymmetric. Owing to this transition, its piezoelectric effect is lost due to the phase transformation providing variation of the elementary crystalline symmetry from asymmetric of FF-PNT to symmetric of FF-PNF. Optical second harmonic generation, which is also related to the lack of symmetry in the native FF-PNT, disappears after thermal treatment due to this crystallographic transition.

Wettability characteristics of FF-PNTs are also strongly affected; the hydrophilic channels of the PNTs are lost after heating, leaving a highly hydrophobic surface. All these properties are irreversibly altered because of the covalent bond that is formed, causing cyclization of the linear peptide, which is the basic building block of the macromolecular structure. The alteration of the building block causes a phase transition in the crystal, which in turn, changes its properties.

Previous studies in our group have found that native FF-PNT can be dissembled into elementary building blocks-peptide nanodots of calibrated size $\sim 2 \mathrm{~nm}$ showing pronounced QC. ${ }^{23}$ The revealed QC effect is well-known for inorganic nanoscale materials but it was never observed in bio-organic nanostructures. ${ }^{21}$ These nanodots are basic units composing these intrinsic supramolecular structures. They have crystalline asymmetry and functional optical, electronic and ferroelectric properties identical to FF-PNT. These allow considering them as self-sustained elementary building blocks and could be used as future new and multifunctional units toward advanced nanotechnological applications, such as biocompatible quantum dot-markers, new generation of quantum dot-lasers, unit cells of ferroelectric memory of ultrahigh density, nanopiezoelectric devices, nonlinear optical convertors at nanoscale and more.

\section{ACKNOWLEDGEMENTS}

This research was supported by a grant from the Ministry of Science \& Technology, Israel \& the Russian Foundation for Basic Research, the Russian Federation.

1 Reches, M. \& Gazit, E. Controlled patterning of aligned self-assembled peptide nanotubes. Nat. Nanotechnol. 1, 195-200 (2006).

2 Ryu, J. \& Park, C. B. High-temperature self-assembly of peptides into vertically well-aligned nanowires by aniline vapor. Adv. Mater. 20, 3754-3758 (2008).

3 Lee, J. S., Yoon, I., Kim, J., Ihee, H., Kim, B. \& Park, C. B. [Self-assembly of semiconducting photoluminescent peptide nanowires in the vapor phase]. Angew. Chem.-Int. Ed 50, 1164-1167 (2011).

4 Adler-Abramovich, L., Aronov, D., Beker, P., Yevnin, M., Stempler, S., Buzhansky, L., Rosenman, G. \& Gazit, E. Vapor-deposited self-assembled peptide nano-array for energy storage and microfluidics devices. Nat. Nanotechnol. 4, 849-854 (2009).

5 Amdursky, N., Beker, P., Koren, I., Bank-Srour, B., Mishina, E., Semin, S., Rasing, T., Rosenberg, Y., Barkay, Z., Gazit, E. \& Rosenman, G. Structural Transition in peptide nanotubes. Biomacromolecules 12, 1349-1354 (2011)

6 Sedman, V. L., Adler-Abramovich, L., Allen, S., Gazit, E. \& Tendler, S. J. B. Direct observation of the release of phenylalanine from diphenylalanine nanotubes. J. Am. Chem. Soc. 128, 6903-6908 (2006).

7 Rosenman, G., Beker, P., Koren, I., Yevnin, M., Bank-Srour, B., Mishina, E. \& Semin, S. Bioinspired peptide nanotubes: deposition technology, basic physics and nanotechnology applications. J. Pept. Sci. 17, 75-87 (2010).

8 Handelman, A., Beker, P., Amdursky, N. \& Rosenman, G. Physics and engineering of peptide supramolecular nanostructures. Phys. Chem. Chem. Phys. 14, 6391-6408 (2012).

9 Gorbitz, C. H. Nanotube formation by hydrophobic dipeptides. Chemistry 7, 5153-5159 (2001).

10 Gorbitz, C. H. The structure of nanotubes formed by diphenylalanine, the core recognition motif of Alzheimer's beta-amyloid polypeptide. Chem. Commun. 22, 2332-2334 (2006)

11 Gorbitz, C. H. Microporous organic materials from hydrophobic dipeptides. Chemistry 13, 1022-1031 (2007).

12 Amdursky, N., Beker, P., Schklovsky, J., Gazit, E. \& Rosenman, G. Ferroelectric and related phenomena in biological and bioinspired nanostructures. Ferroelectrics 399 107-117 (2010)

13 Kholkin, A., Amdursky, N., Bdikin, I., Gazit, E. \& Rosenman, G. Strong piezoelectricity in bioinspired peptide nanotubes. ACS nano. 4, 610-614 (2009). 
14 Amdursky, N., Gazit, E. \& Rosenman, G. Quantum confinement in self-assembled bioinspired peptide hydrogels. Adv. Mater. 22, 2311-2315 (2010).

15 Kumaraswamy, P., Lakshmanan, R., Sethuraman, S. \& Krishnan, U. M. Self-assembly of peptides: influence of substrate, $\mathrm{pH}$ and medium on the formation of supramolecular assemblies. Soft Matter 7, 2744-2754 (2011).

16 Abbott, J. P. \& Zeina, J. J. Vacuum technology considerations for mass metrology. J. Res. Natl. Inst. Stand. Technol. 116, 689-702 (2011).

17 Reches, M. \& Gazit, E. Casting metal nanowires within discrete self-assembled peptide nanotubes. Science 300, 625-627 (2003).

18 Kol, N., Adler-Abramovich, L., Barlam, D., Shneck, R. Z., Gazit, E. \& Rousso, I. Selfassembled peptide nanotubes are uniquely rigid bioinspired supramolecular structures. Nano. Lett. 5, 1343-1346 (2005).
19 Amdursky, N., Molotskii, M., Aronov, D., Adler-Abramovich, L., Gazit, E. \& Rosenman, G. Blue luminescence based on quantum confinement at peptide nanotubes. Nano Lett. 9, 3111-3115 (2009).

20 Amdursky, N., Molotskii, M., Gazit, E. \& Rosenman, G. Self-assembled bioinspired quantum dots: optical properties. Appl. Phys. Lett. 94, 261907 (2009).

21 Hauser, C. A. E. \& Zhang, S. G. Nanotechnology: peptides as biological semiconductors. Nature 468, 516-517 (2010).

22 Amdursky, N., Koren, I., Gazit, E. \& Rosenman, G. Adjustable Photoluminescence of peptide nanotubes coatings. J. Nanosci. Nanotechnol. 11, 9282-9286 (2011).

23 Amdursky, N., Molotskii, M., Gazit, E. \& Rosenman, G. Elementary building blocks of self-assembled peptide nanotubes. J. Am. Chem. Soc. 132, 15632-15636 (2005). 